\title{
Some Relations between Certain Complex Equations and Nonnormalized Meromorphic Functions
}

\author{
Hüseyin Irmak \\ Department of Mathematics, Faculty of Science, Çankırı Karatekin University, 18100 Çankırı, Turkey \\ Correspondence should be addressed to Hüseyin Irmak; hisimya@yahoo.com
}

Received 28 December 2013; Accepted 20 March 2014; Published 8 April 2014

Academic Editor: Feng Feng

Copyright (C) 2014 Hüseyin Irmak. This is an open access article distributed under the Creative Commons Attribution License, which permits unrestricted use, distribution, and reproduction in any medium, provided the original work is properly cited.

The purpose of this investigation is first to reveal some relations between certain complex (differential) equations and nonnormalized meromorphic functions and then to point some of their useful consequences out.

\section{Introduction, Notations, Definitions, and Motivation}

As is known, a differential equation is an equation that involves the derivatives of a function as well as the function itself. If partial derivatives are involved, the equation is called a partial differential equation; if only ordinary derivatives are present, the equation is called an ordinary differential equation (ODE). Differential equations play an extremely important and useful role in applied math, engineering, and physics, and more mathematical and numerical machineries have been developed for the solution to differential equations.

As we also know, ODE is a differential equation in which the unknown function (also known as the dependent variable) is a function of a single independent variable. In the simplest form, the unknown function is a real- or complexvalued function, but more generally, it may be vector-valued or matrix-valued; this corresponds to considering a system of ordinary differential equations for a single function. Ordinary differential equations are further classified according to the order of the highest derivative of the dependent variable with respect to the independent variable appearing in the equation. The most important cases for applications are first-order and second-order differential equations.

The theory of differential equations is closely related to the theory of difference equations, in which the coordinates assume only discrete values, and the relationship involves values of the unknown function or functions and values at nearby coordinates. There are many methods to compute numerical solutions to differential equations and studies on the properties of differential equations involving approximation theory of the solution to a differential equation by the solution to a corresponding differential equation.

In this work, as a novel investigation, we want to focus on certain types of (linear or nonlinear) first-order complex differential equation. Especially, for functions $A(z)$ and $B(z)$ being analytic in certain domains of complex domain, we want to take cognizance of a few types of certain complex equations including several first-order complex differential equations like

$$
A(z) W^{\prime}(z)+B(z) W(z)+C(z)=0
$$

and to reveal some of their useful implications between certain complex (differential) equations and nonnormalized multivalent functions which are analytic in the punctured unit disk and meromorphic in the unit disk. For those, we need to present some information and introduce certain definitions.

First of all, here and throughout this paper, let $\mathbb{R}$ be the set of real numbers, let $\mathbb{C}$ be the set of complex numbers, let $\mathbb{N}$ be the set of positive integers, let $\mathbb{U}$ be the open unit disk, and let $\mathbb{D}$ be the punctured unit disk in $\mathbb{C}$, that is, $\mathbb{U}=\{z: z \in \mathbb{C}$ and $|z|<1\}$ and $\mathbb{D}:=\mathbb{U}-\{0\}$. Let also $\mathscr{M}_{p, q}^{\kappa}$ denote the family consisting of the nonnormalized meromorphically multivalent functions $f(z)$ in the following form:

$$
\begin{array}{r}
f(z)=\frac{\kappa}{z^{q}}+\frac{a_{p}}{z^{q-p}}+\frac{a_{p+1}}{z^{q-p-1}}+\frac{a_{p+2}}{z^{q-p-2}}+\cdots+\frac{a_{p+n}}{z^{q-p-n}}+\cdots \\
\left(\kappa \neq 0 ; p \in \mathbb{N} ; q \in \mathbb{N} ; n \in \mathbb{N} \cup\{0\} ; a_{k} \in \mathbb{C} ; z \in \mathbb{D}\right),
\end{array}
$$


which are analytic in the domain $\mathbb{D}$ and which (in the special case when $q=1)$ have a simple pole at origin $(z=0)$ with residue $\kappa$ there.

By making use of the function $f(z)$ belonging to the class $\mathscr{M}_{p, q}^{\kappa}$, which is, of course, in the form of (2), we now define three operators which are $\mathscr{L}_{z}^{n}[\cdot], \mathscr{D}_{z}^{n}[\cdot]$, and $\mathscr{W}_{m, n}[f]$, respectively, in the following forms:

$$
\begin{gathered}
\mathscr{L}_{z}^{n}[f] \equiv \mathscr{L}_{z}^{n}[f(z)] \equiv \frac{d^{n}}{d z^{n}}[f(z)]=\frac{d^{n} f}{d z^{n}}, \\
\mathscr{D}_{z}^{n}[g] \equiv \mathscr{D}_{z}^{n}[g(z)] \equiv \frac{d^{n}}{d z^{n}}[g(z)]=\frac{d^{n} g}{d z^{n}} \\
\left(g(z):=z^{n+2 q} \mathscr{L}_{z}^{n}\{f(z)\}\right), \\
\mathscr{W}_{m, n}[f] \equiv \mathscr{W}_{m, n}[f(z)] \equiv \frac{z \mathscr{D}_{z}^{1+m}\left\{z^{n+2 q} \mathscr{L}_{z}^{n}\{f(z)\}\right\}}{\mathscr{D}_{z}^{m}\left\{z^{n+2 q} \mathscr{L}_{z}^{n}\{f(z)\}\right\}},
\end{gathered}
$$

where $p \in \mathbb{N}, q \in \mathbb{N}, n \in \mathbb{N}_{0}:=\mathbb{N} \cup\{0\}, m \in \mathbb{N}_{0}$, and $z \in \mathbb{D}$.

As we have said shortly before, the purpose of this present paper is first to determine some operators generated by the nonnormalized meromorphically multivalent functions and, in view of them, to characterize some complex (differential) equations and then to reveal some useful results between the related equations and nonnormalized meromorphic functions and also to point a number of their consequences relating to univalent functions out. For the proofs of the main results, one of the novel types of the well-known assertions generated by Miller and Mocanu in [1, pages 33-35] which are more general than the result of Jack [2] is used there. In addition, for researchers who have been working on these topics, some works dealing with the related proof techniques in $[3,4]$ and also in [5] can be checked, as examples.

We now recall the following assertions that will be required in the present research.

Lemma 1 (see[1]). Let $\Omega \subset \mathbb{C}$ and suppose that the function $\psi: \mathbb{C}^{2} \times \mathbb{U} \rightarrow \mathbb{C}$ satisfies $\psi\left(M e^{i \theta}, K e^{i \theta} ; z\right) \notin \Omega$ for all $K \geq l \cdot M$, $\theta \in \mathbb{R}$, and $z \in \mathbb{U}$. If the function $p(z)$ is in the class $\mathscr{H}[b, l] \equiv$ $\left\{p(z) \in \mathscr{H}(\mathbb{U}): p(z)=b+b_{l} z^{l}+\cdots(l \in \mathbb{N}, z \in \mathbb{U})\right\}$ and $\psi\left(p(z), z p^{\prime}(z) ; z\right) \in \Omega$ for all $z \in \mathbb{U}$, then $|p(z)|<M(z \in \mathbb{U})$.

Lemma 2 (see [1]). Let $\Omega \subset \mathbb{C}$ and suppose that the function $\psi: \mathbb{C}^{2} \times \mathbb{U} \rightarrow \mathbb{C}$ satisfies $\psi(i x, y ; z) \notin \Omega$ for all $x \in \mathbb{R}$, $y \leq-l\left(1+x^{2}\right) / 2$, and $z \in \mathbb{U}$. If the function is $p \in \mathscr{H}[b, l]$, and $\psi\left(p(z), z p^{\prime}(z) ; z\right) \in \Omega$ for all $z \in \mathbb{U}$, then $\mathfrak{R} e\{p(z)\}>$ $0(z \in \mathbb{U})$.

\section{The Main Results}

Before proving the main results, it will be to the point that we derive the open forms of the applications of the operators defined by (3) and (4). For this reason, by applying the related operators to the functions stated in (3) and (4), respectively, the following expressions are easily derived:

$$
\begin{aligned}
& \mathscr{L}_{z}^{n}[f] \equiv \frac{(q+n-1) !}{(q-1) !} \cdot \frac{\kappa}{z^{n+q}}(-1)^{n}+\sum_{k=p}^{\infty} \frac{k !}{(k-n) !} a_{k} z^{k-n}, \\
& \mathscr{D}_{z}^{m}\left[z^{n+2 q} \mathscr{L}_{z}^{n}[f]\right] \equiv \frac{q(q+n-1) !}{(q-m) !} \cdot \frac{\kappa}{z^{m-q}}(-1)^{n} \\
&+\sum_{k=p}^{\infty} \frac{k !(k+2 q) !}{(k-n) !(k+2 q-m) !} a_{k} z^{k+2 q-m},
\end{aligned}
$$

where, for all $\kappa \neq 0, p>n, q>m, p \in \mathbb{N}, q \in \mathbb{N}, n \in \mathbb{N}_{0}$, and $m \in \mathbb{N}_{0}$.

We now begin by setting and then by proving the following theorems concerning certain results derived by using both the above operators together with (4) and Lemma 1.

Theorem 3. Let $M>0, l \in \mathbb{N}, \gamma \in \mathbb{C}^{*}:=\mathbb{C}-\{0\}$, and let the function $\Psi(z)$ satisfy the inequality

$$
\mathfrak{R} e(\Psi(z))<\frac{l \cdot M}{1+M} \quad(z \in \mathbb{U})
$$

and also let the function $f(z) \in \mathscr{M}_{p, q}^{\kappa}$ be the (unique) solution to the complex equation

$$
\gamma \cdot\left(\mathscr{W}_{m, n}[f]\right)^{\prime}-\Psi(z) \cdot\left(\mathscr{W}_{m, n}[f]\right)=0 \quad(z \in \mathbb{D}) .
$$

Then,

$$
\left|\left(\frac{\mathscr{W}_{m, n}[f]}{q-m}\right)^{\gamma}-1\right|<M \quad\left(M>0 ; z \in \mathbb{D} ; \gamma \in \mathbb{C}^{*}\right),
$$

where the value of the above complex power is taken to be as its principal value and the operator $\mathscr{W}_{m, n}[f](z)$ is defined by (5).

Proof. In light of (6) and also (5), let us define a function $p(z)$ by

$$
p(z)=\left(\frac{\mathscr{W}_{m, n}[f]}{q-m}\right)^{\gamma}-1 \quad\left(\gamma \in \mathbb{C}^{*} ; f(z) \in \mathscr{M}_{p, q}^{\kappa}\right) .
$$

Clearly, $p(z)$ is both an analytic function in $\mathbb{D}$ and meromorphic function in $\mathbb{U}$, and also it is in the class $\mathscr{H}[0, l](l \in \mathbb{N})$, which is defined as in Lemma 1 with $b=0$, and

$$
\Psi(z):=\frac{z p^{\prime}(z)}{1+p(z)}=\gamma \cdot \frac{z\left\{\mathscr{W}_{m, n}[f]\right\}^{\prime}}{\mathscr{W}_{m, n}[f]} .
$$

It is easily seen that the function $\Psi(z)$ is an analytic function in the domain $\mathbb{U}$ and a solution to the complex equation given by $(8)$. 
Let

$$
\begin{gathered}
\frac{z p^{\prime}(z)}{1+p(z)}=\gamma \cdot \frac{z\left\{\mathscr{W}_{m, n}[f]\right\}^{\prime}}{\mathscr{W}_{m, n}[f]}=: \mathscr{V}_{n}[m, \gamma ; f](z), \\
\psi(r, s ; z)=\frac{s}{1+r}, \quad \Omega=\left\{w: w \in \mathbb{C}, \mathfrak{R} e\{w\}<\frac{l M}{1+M}\right\} .
\end{gathered}
$$

Then it is easily observed that

$$
\psi\left(p(z), z p^{\prime}(z) ; z\right)=\mathscr{V}_{n}[m, \gamma ; f](z) \in \Omega
$$

for all $z \in \mathbb{U}$. In addition, for any $\theta \in \mathbb{R}, K>l M$, and $z \in \mathbb{U}$, due to $M>0$ we arrive at

$$
\mathfrak{R} e\left\{\psi\left(M e^{i \theta}, K e^{i \theta} ; z\right)\right\}=\mathfrak{R} e\left(\frac{K e^{i \theta}}{1+M e^{i \theta}}\right) \geq \frac{l M}{1+M},
$$

that is, $\psi\left(M e^{i \theta}, K e^{i \theta} ; z\right) \notin \Omega$. Therefore, in view of Lemma 1, the assumption in (10) yields that

$$
|p(z)|=\left|\left(\frac{\mathscr{W}_{m, n}[f]}{q-m}\right)^{\gamma}-1\right|<M \quad(z \in \mathbb{D} ; M>0),
$$

which is the proof of Theorem 4 .

With the help of the related operators and Lemma 2, the second theorem (below) can be also proved.

Theorem 4. Let $0 \leq \mu<1, l \in \mathbb{N}, \gamma \in \mathbb{C}^{*}, z \in \mathbb{D}$, and let the function $\Phi(z)$ satisfy any of the inequalities

$$
\mathfrak{R} e(\Phi(z))> \begin{cases}\frac{l \mu}{2(\mu-1)} & \text { if } 0 \leq \mu \leq \frac{1}{2} \\ \frac{l(\mu-1)}{2 \mu} & \text { if } \frac{1}{2} \leq \mu<1\end{cases}
$$

and also let the function $f(z) \in \mathscr{M}_{p, q}^{\kappa}$ be the solution to the complex equation given by (8). Then,

$$
\mathfrak{R} e\left\{\left(\frac{\mathscr{W}_{m, n}[f]}{q-m}\right)^{\gamma}\right\}>\mu
$$

where the value of the above complex expression is taken to be as its principal value and the operator $\mathscr{W}_{m, n}[f](z)$ is defined by (5).

Proof. With the help of (6) and also (5), again define a function $p(z)$ in the following form:

$$
\begin{array}{r}
p(z)=\frac{\left(\mathscr{W}_{m, n}[f] /(q-m)\right)^{\gamma}-\mu}{1-\mu} \\
\left(0 \leq \mu<1 ; f(z) \in \mathscr{M}_{p, q}^{\kappa}\right) .
\end{array}
$$

Then, it is easy to see that $p(z)$ is both an analytic function in $\mathbb{D}$ and meromorphic function in $\mathbb{U}$. Further, $p(z)$ belongs to the class $\mathscr{H}[1, l]$, which is the class given in Lemma 1 with $b=1$. The equality in (18) immediately yields that

$$
\begin{array}{r}
\frac{(1-\mu) z p^{\prime}(z)}{\mu+(1-\mu) p(z)}=\gamma \cdot \frac{z\left(\mathscr{W}_{m, n}[f]\right)^{\prime}}{\mathscr{W}_{m, n}[f]}=: \Phi(z) \\
(0 \leq \mu<1 ; z \in \mathbb{U}) .
\end{array}
$$

It is clear that $\Phi(z)$ is an analytic function in the disk $\mathbb{U}$ and also the solution to the equation given by (8).

Let

$$
\begin{array}{r}
\frac{(1-\mu) z p^{\prime}(z)}{\mu+(1-\mu) p(z)}=\gamma \cdot \frac{z\left(\mathscr{W}_{m, n}[f]\right)^{\prime}}{\mathscr{W}_{m, n}[f]}=: \mho_{n}[m ; \gamma ; f](z) \\
(0 \leq \mu<1 ; z \in \mathbb{U}) .
\end{array}
$$

Then, from the following definitions:

$$
\begin{aligned}
& \psi(r, s ; z)=\frac{(1-\mu) s}{\mu+(1-\mu) r} \quad(0 \leq \mu<1), \\
& \Omega=\{w: w \in \mathbb{C}, \\
& \mathfrak{R e}\{w\}>\left\{\begin{array}{ll}
\frac{l \mu}{2(\mu-1)} & \text { when } \mu \in\left[0, \frac{1}{2}\right] \\
\frac{l(\mu-1)}{2 \mu} & \text { when } \mu \in\left[\frac{1}{2}, 1\right)
\end{array}\right\},
\end{aligned}
$$

it can be easily seen that

$$
\psi\left(p(z), z p^{\prime}(z) ; z\right)=\mho_{n}[m, \gamma ; f](z) \in \Omega
$$

for all $z \in \mathbb{U}$. Further, for any

$$
x \in \mathbb{R}, \quad y \leq-\frac{l\left(1+x^{2}\right)}{2}, \quad l \in \mathbb{N}, z \in \mathbb{U},
$$

and owing to

$$
\begin{aligned}
& 0 \leq \mu \leq \frac{1}{2} \Longrightarrow \frac{1+x^{2}}{(\mu /(1-\mu))^{2}+x^{2}} \geq 1, \\
& \frac{1}{2} \leq \mu<1 \Longrightarrow \frac{1+x^{2}}{1+((1-\mu) / \mu)^{2} x^{2}} \geq 1,
\end{aligned}
$$

we so get that

$$
\begin{aligned}
\mathfrak{R} e\{\psi(i x, y ; z)\}= & \frac{(1-\mu) y}{(1-\mu)^{2} x^{2}+\mu^{2}} \\
& \leq \begin{cases}\frac{l \mu(\mu-1)}{2} \cdot \frac{1}{(1-\mu)^{2}} & \text { if } \mu \in\left[0, \frac{1}{2}\right] \\
\frac{l \mu(\mu-1)}{2} \cdot \frac{1}{\mu^{2}} & \text { if } \mu \in\left[\frac{1}{2}, 1\right) ;\end{cases}
\end{aligned}
$$

that is, $\psi(i x, y ; z)$ does not belong to $\Omega$. 
Finally, from (18), we obtain the inequality

$$
\begin{array}{r}
\mathfrak{R} e\{p(z)\}=\mathfrak{R} e\left\{\frac{\left(\mathscr{W}_{m, n}[f] /(q-m)\right)^{\gamma}-\mu}{1-\mu}\right\}>0 \\
(z \in \mathbb{D} ; 0 \leq \mu<1),
\end{array}
$$

which implies the inequality in (17). This completes the proof of Theorem 4 .

\section{Certain Implications of the Main Results}

The operators defined by (3), (4), and (5) have a major contribution to both analytic and geometric function theory (cf., e.g., [6-9]), as their certain conclusions. For these, selecting the appropriate values of the parameters in the definition of $\mathscr{W}_{m, n}[f]$, several rational type functions are easily reserved. For example, the following relations can be considered:

$$
\begin{aligned}
m=n=0 \Longrightarrow \mathscr{W}_{0,0}[f] & \equiv \frac{z_{z}^{1+0}\left\{z^{0+2 q} \mathscr{L}_{z}^{0}\{f(z)\}\right\}}{\mathscr{D}_{z}^{0}\left\{z^{0+2 q} \mathscr{L}_{z}^{0}\{f(z)\}\right\}} \\
& =2 q+\frac{z f^{\prime}(z)}{f(z)} \\
m=n-1=0 \Longrightarrow \mathscr{W}_{0,1}[f] & \equiv \frac{\mathscr{D}_{z}^{1+0}\left\{z^{1+2 q} \mathscr{L}_{z}^{1}\{f(z)\}\right\}}{\mathscr{D}_{z}^{0}\left\{z^{1+2 q} \mathscr{L}_{z}^{1}\{f(z)\}\right\}} \\
& =1+2 q+\frac{z f^{\prime \prime}(z)}{f^{\prime}(z)},
\end{aligned}
$$

where $f(z) \in \mathscr{M}_{p, q}^{\kappa}$ and $z \in \mathbb{D}$. Clearly, the above rational type functions are both multivalently analytic in $\mathbb{U}$ and multivalently meromorphic in $\mathbb{D}$. Indeed, in statements like (27) and also (5), in which the functions quotients involved have removable singularities at the point $z=0$, it is tacitly assumed that such function quotients have had these singularities removed. Thereby, those also are important results for geometric function theory (cf., e.g., $[6,7]$ and also see, for certain operators, [10-12]). In addition, if one focuses on the main results, several useful consequences related to both Theorems 3 and 4 can be easily revealed. Some of them deal with analytic function theory and others are related to geometric function theory. In this chapter, only two consequences belonging to two theorems were indicated below. To reveal the other possible consequences (which will be also interested in analytic or geometric properties of complex function with one variable), it is possible to choose the suitable values of the parameters in all theorems (or in their special results). Although these are omitted here, researchers who have been working on this area may easily reveal them.

By setting $n:=0, m:=0$, and $\delta:=\gamma \in \mathbb{R}^{*}:=\mathbb{R}-\{0\}$ in Theorem 3 , the following proposition which also includes meromorphic starlikeness in the domain $\mathbb{U}$ (or in $\mathbb{D}$ ) can be firstly proven.
Proposition 5. Let $M>0, l \in \mathbb{N}, q \in \mathbb{N}, \delta \in \mathbb{R}^{*}$, and let the function $\Theta(z)$ satisfy any of the following inequalities:

$$
\mathfrak{R e}(\Theta(z)) \begin{cases}<\frac{l M}{\delta(1+M)} & \text { when } \delta>0 \\ >\frac{l M}{\delta(1+M)} & \text { when } \delta<0\end{cases}
$$

and also let the function $w:=f(z) \in \mathscr{M}_{p, q}^{\kappa}$ be the solution to the following complex differential equation:

$$
z^{2} w w^{\prime \prime}+z(w-\Theta(z)) w^{\prime}-z^{2}\left(w^{\prime}\right)^{2}+2 q \Theta(z) w=0 .
$$

Then

$$
\left|\left(2 q+\frac{z w^{\prime}(z)}{w(z)}\right)^{\delta}-q^{\delta}\right|<M q^{\delta}
$$

holds, where the value of the complex power above is taken into account to be as its principal value.

By taking $n:=1, m:=0$, and $\delta:=\gamma \in \mathbb{R}^{*}$ in Theorem 4 , the following proposition which also contains meromorphic convexity in the domain $\mathbb{U}$ (or in $\mathbb{D}$ ) can be then proved.

Proposition 6. Let $M>0, l \in \mathbb{N}, q \in \mathbb{N}, \delta \in \mathbb{R}^{*}$, and let the function $\Theta(z)$ satisfy any of the following inequalities:

$$
\operatorname{Re}(\Theta(z)) \begin{cases}>\frac{l \mu}{2 \delta(\mu-1)} & \text { when } \delta>0, \mu \in\left[0, \frac{1}{2}\right] \\ >\frac{l(\mu-1)}{2 \delta \mu} & \text { when } \delta>0, \mu \in\left[\frac{1}{2}, 1\right) \\ <\frac{l \mu}{2 \delta(\mu-1)} & \text { when } \delta<0, \mu \in\left[0, \frac{1}{2}\right] \\ <\frac{l(\mu-1)}{2 \delta \mu} & \text { when } \delta<0, \mu \in\left[\frac{1}{2}, 1\right)\end{cases}
$$

and also let the function $w:=f(z) \in \mathscr{M}_{p, q}^{\kappa}$ be the solution to the following complex differential equation:

$$
\begin{aligned}
z^{2} w^{\prime} w^{\prime \prime \prime} & +z\left(w^{\prime}-\Theta(z)\right) w^{\prime \prime}-z^{2}\left(w^{\prime \prime}\right)^{2} \\
& +(1+2 q \Theta(z)) w^{\prime}=0 .
\end{aligned}
$$

Then

$$
\mathfrak{R} e\left[\left(1+2 q+\frac{z w^{\prime \prime}(z)}{w^{\prime}(z)}\right)^{\delta}\right]>\mu \quad(0 \leq \mu<1)
$$

is true, where the value of the complex power above is taken into consideration to be as its principal value.

\section{Conflict of Interests}

The author declares that there is no conflict of interests regarding the publication of this paper. 


\section{Acknowledgment}

The work on this study was supported by the International Research Project financed by TÜBITAK (The Scientific and Technical Research Council of TURKEY) with the Project no. U-105T056.

\section{References}

[1] S. S. Miller and P. T. Mocanu, Differential Subordinations, Theory and Applications, Monographs and Textbooks in Pure and Applied Mathematics, Marcel Dekker, New York, NY, USA, 2000.

[2] I. S. Jack, "Functions starlike and convex of order $\alpha$," Journal of the London Mathematical Society, vol. 3, pp. 469-474, 1971.

[3] H. Irmak and M. San, "Some relations between certain inequalities concerning analytic and univalent functions," Applied Mathematics Letters, vol. 23, no. 8, pp. 897-901, 2010.

[4] H. Irmak, "Some relationships between certain multivalently analytic and multivalently meromorphic functions," Mathematical and Computer Modelling, vol. 57, no. 3-4, pp. 564-569, 2013.

[5] H. Irmak, T. Bulboacă, and N. Tuneski, "Some relations between certain classes consisting of $\alpha$-convex type and Bazilević type functions," Applied Mathematics Letters, vol. 24, no. 12, pp. $2010-$ 2014, 2011.

[6] P. L. Duren, Univalent Functions, vol. 259 of Grundlehren der Mathematischen Wissenschaften, Springer, New York, NY, USA, 1983.

[7] A. W. Goodman, Univalent Functions, vol. 1-2, Polygonal Publishing House, Washington, NJ, USA, 1983.

[8] Z. Nehari, Conformal Mapping, McGraw-Hill Book Company, New York, NY, USA, 1952.

[9] C. Pommerenke, Univalent Functions, Studia Mathematica, Bd. 25, Vandenhoeck and Ruprecht, Göttingen, Germany, 1952.

[10] H. Irmak and R. K. Raina, "New classes of non-normalized meromorphically multivalent functions," Sarajevo Journal of Mathematics, vol. 3, no. 2, pp. 157-162, 2007.

[11] H. Irmak, N. E. Cho, Ö. F. Çetin, and R. K. Raina, "Certain inequalities involving meromorphically multivalent functions," Hacettepe Bulletin of Natural Sciences and Engineering B: Mathematics and Statistics, vol. 30, pp. 39-43, 2001.

[12] H. Irmak, G. Tinaztepe, N. Tuneski, and M. Şan, "An ordinary differential operator and its applications to certain classes of multivalently meromorphic functions," Bulletin of Mathematical Analysis and Applications, vol. 1, no. 2, pp. 17-22, 2009. 


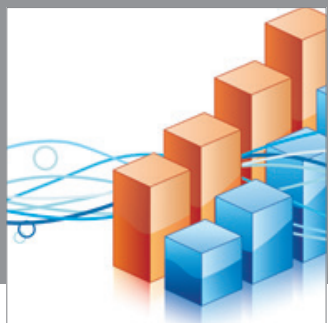

Advances in

Operations Research

mansans

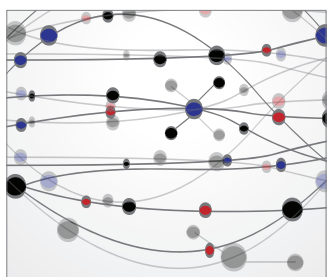

The Scientific World Journal
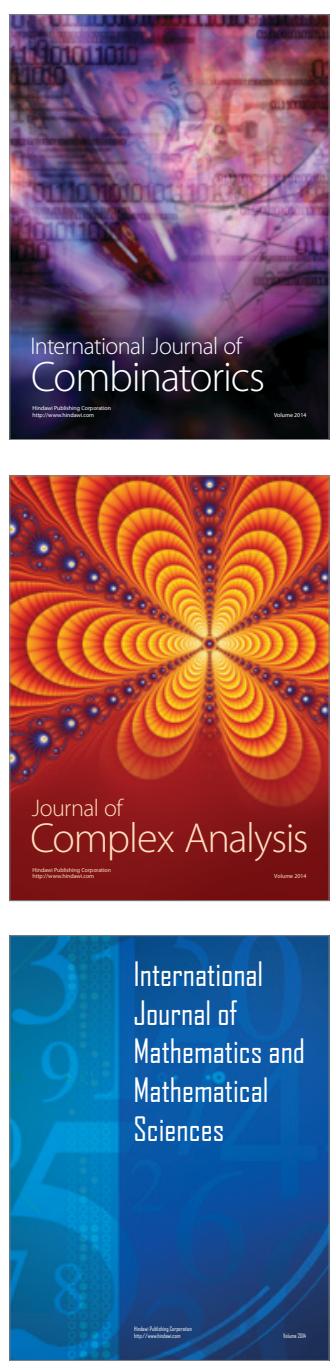
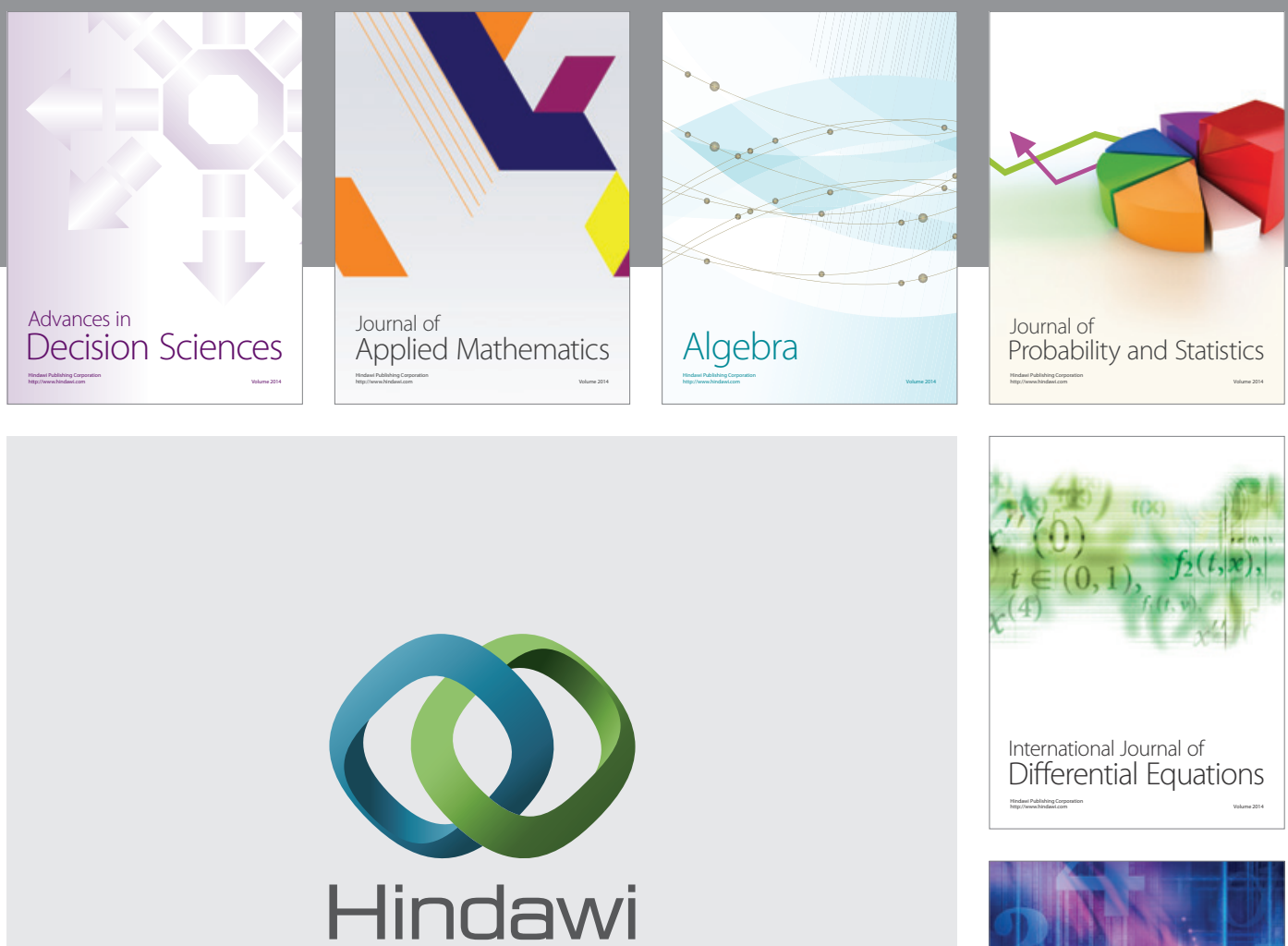

Submit your manuscripts at http://www.hindawi.com
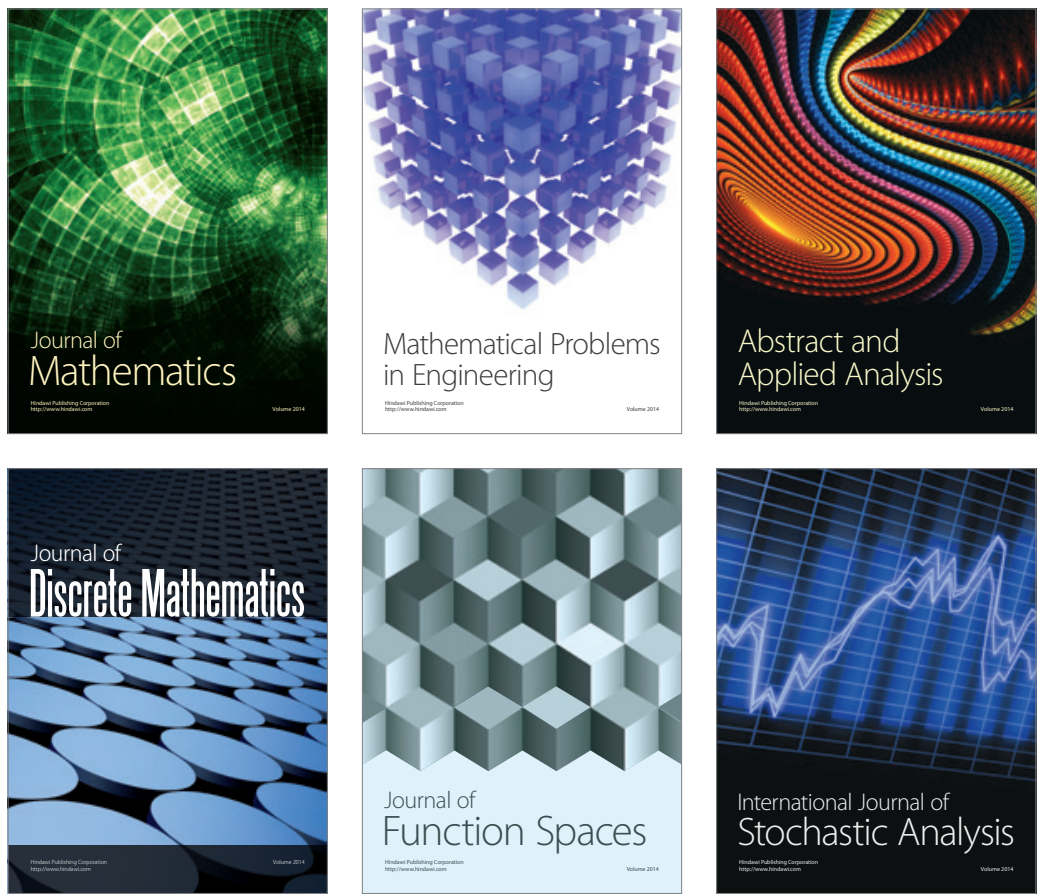

Journal of

Function Spaces

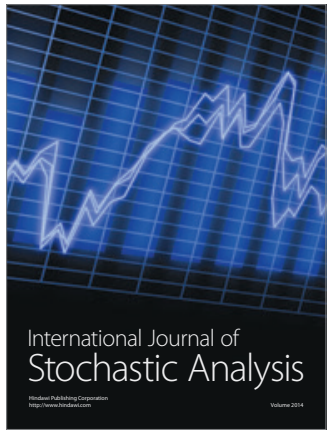

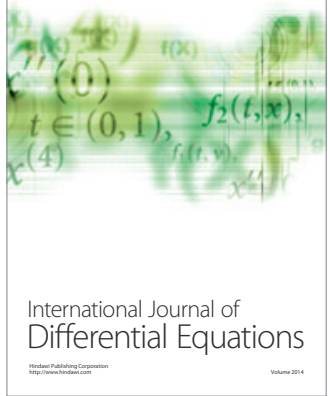
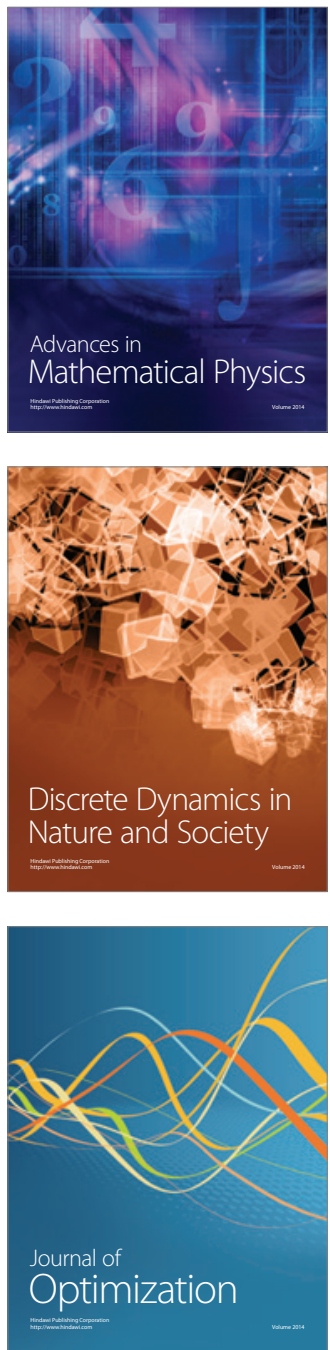